\title{
Extended Depth of Focus intraocular lenses - the present and future in cataract surgery
}

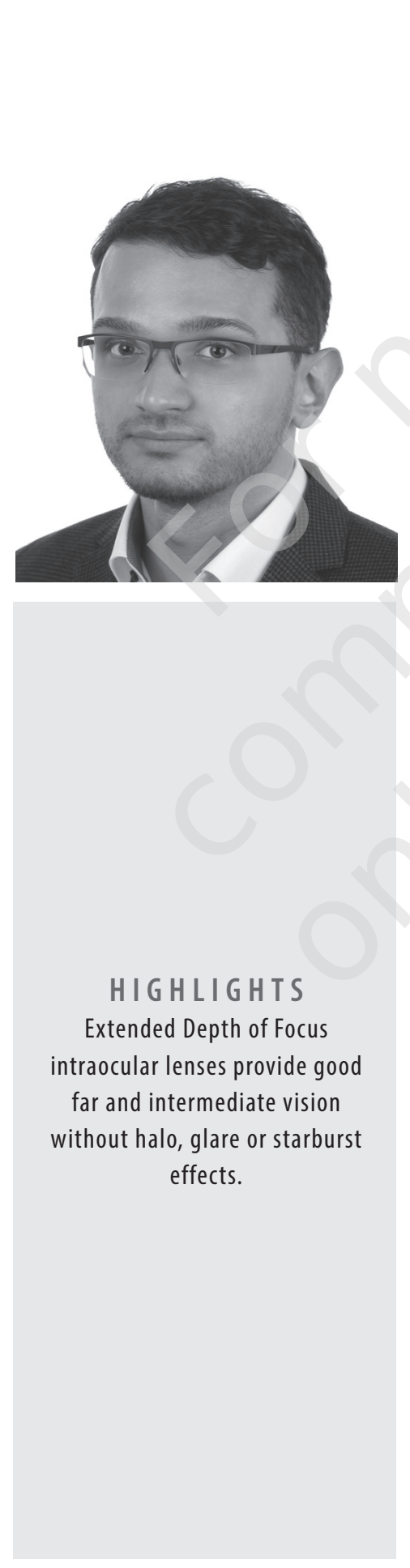

\section{Andrzej Grąbczewski ${ }^{1},{ }^{2}$, Martyna Jendrzejczyk ${ }^{1}$}

${ }^{1}$ Independent Public Clinical Ophthalmology Hospital, Warsaw, Poland Head: Prof. Jacek P. Szaflik, MD, PhD

2"Świat Oka"Ophthalmological Center Scientific director: Anna M. Ambroziak, MD, PhD Medical director: Joanna Gołębiewska, MD, PhD

\section{ABSTRACT}

The purpose of this article is to evaluate the technology behind intraocular lenses with extended depth of focus, explain how they work, assess their benefits for patients, and summarize our own early experience.

Key words: intraocular lens, Extended Depth of Focus lenses 


\section{INTRODUCTION}

The Extended Depth of Focus intraocular lenses (EDoF IOLs) are considerably new, heterogenous group of intraocular lenses with properties that seem to respond well to the visual patients' needs and provide good postoperative outcomes. The first EDoF lens was released in 2014 (Symfony, Johnson \& Johnson). Since then, new lenses that use new technologies and have new operating principles have regularly emerged. EDoF lenses provide good vision at distance, similar to monofocal lenses [1], and better vision at intermediate distances in comparison to monofocal and multifocal lenses [2]. Near vision has also been very satisfactory in most patients [3]. Our experience shows that patients do not require additional correction to their glasses for most of their daily activities. EDoF lenses can be used for both refractive lens replacement and cataract surgery. One very significant cause of the deterioration of the quality of vision in Europe [4] and in the world [5] is presbyopia. This insufficiency is also responsible for a significant deterioration of the quality of life $[6,7]$. There is a great need to improve the quality of life of patients using a safe method that does not cause adverse effects. Due to aging of the population, cataracts are another very common cause of the deterioration of vision and quality of life [7]. The use of well-tolerated EDoF lenses is an opportunity to improve the quality of vision and life in both group of people.

\section{DEFINITION OF EDOF LENSES AND THEIR FUNCTIONING}

EDoF lenses are a heterogeneous group of intraocular lenses. There are many types of EDoF lenses available on the market, from various manufacturers, including Zeiss AT LARA, Alcon Vivity, TECNIS Eyhance, TECNIS Symfony, Swiss Advanced Vision Lucidis, and SIFI Mini Well Ready. The construction and technology of the lenses differ from each other, depending on the individual company. However, the operating principles are always the same and are based on the creation of pseudo-accommodation through the use of a single, Extended Depth of Focus, that gives a greater depth of field [8] and better vision over intermediate distances [2] while still maintaining good distance vision [1] (fig. 1).

\section{FIGURE ( 1}

\section{Extended Depth of Focus lenses - principle of operation.}

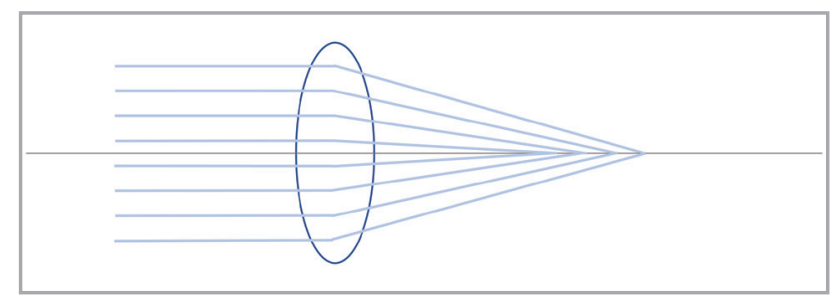

EDoF lenses work based on designed imperfections in the lens, which deform the images received by the wearer. In most cases, undesirable higher-order aberrations, such as spherical aberrations, or comas, are responsible for the depth of field in EDoF lenses.

A characteristic feature of a lens or optical system is spherical aberration, which refers to the differing refraction of light beams, and as a result, different focal lengths, due to the location of the aberration between the center and the edge of the lens (fig. 2). Monofocal lenses use aspherical lenses, which produce a clearer image without distortions for monofocal lenses, for example, for correcting far-sightedness.

\section{FIGURE 2}

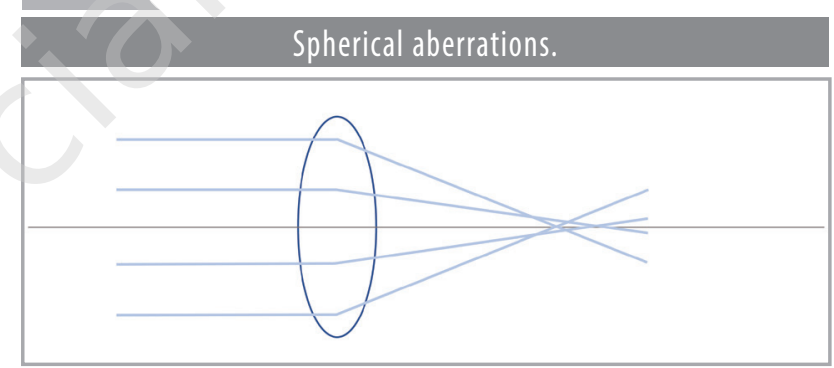

Another characteristic feature of a lens or optical system is chromatic aberration, which is the different focusing of a light wave due to the different refractive indexes for different light wavelengths (fig. 3). Chromatic aberrations cause lower contrast in vision. Consequently, achromaticity does not improve a lens's depth of field, but by improving contrast it does improve visual quality [9].

\section{FIGURE $(3$}

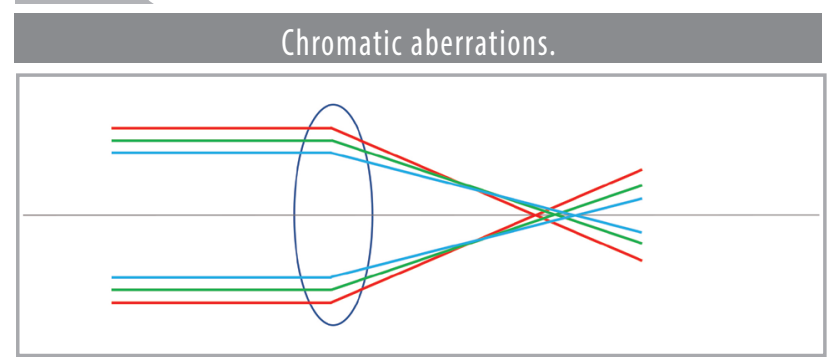

Another phenomenon is a pinhole effect that eliminates rays from the light beam that do not pass through the pinhole perfectly perpendicularly (fig. 4). This largely prevents the formation of undesirable spherical aberrations. Physiologically, this effect is responsible for poorer vision in scotopic conditions and provides greater depth of field but with a reduction in field of view, due to constriction of the pupil. 


\section{FIGURE 4}

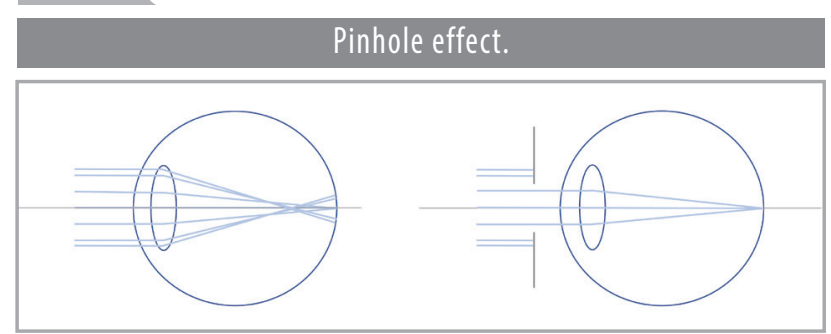

By definition, EDoF lenses should make use of spherical aberrations and can additionally use the stenopeic hole effect to obtain a smooth, Extended Depth of Focus, and improved depth of field. Some authors suggest that diffractive lenses, or lenses that use additional chromatic aberrations are not pure EDoF lenses, and recommend calling them hybrid lenses [8].

The aim of Extended Depth of Focus is to eliminate the overlapping of images from the part of the lens responsible for vision at distance, and the part responsible for vision at intermediate distance [9], which occurs in multifocal lenses. The use of non-diffractive, Extended Depth of Focus through a smooth transition between optical zones does not result in the splitting of light rays at the zone boundary, thus reducing or completely eliminating positive dysphotopsia such as halos, glare, and starbursts. This is, in addition to better contrast vision, the greatest advantage of extended depth-offocus lenses compared to multifocal lenses.

One of the EDoF lenses that is available in Poland is the AT LARA by ZEISS (fig. 5). This is an aspherical intraocular lens that comes preloaded into the injector. The Extended Depth of Focus in the lens is achieved thanks to diffraction rings connected by the so-called transition zones. This technology is called Smooth Microphase (SMP). The transition zones provide a much smoother transition between the diffraction rings, which ensures less splitting of the light beam at the zone boundary and reduces unwanted lighting effects. According to the manufacturer, the lens provides good vision of 0.8 or more without correction to a distance of $55 \mathrm{~cm}$, and 0.63 without correction to a distance of $45 \mathrm{~cm}$.

\section{FIGURE ( 5}

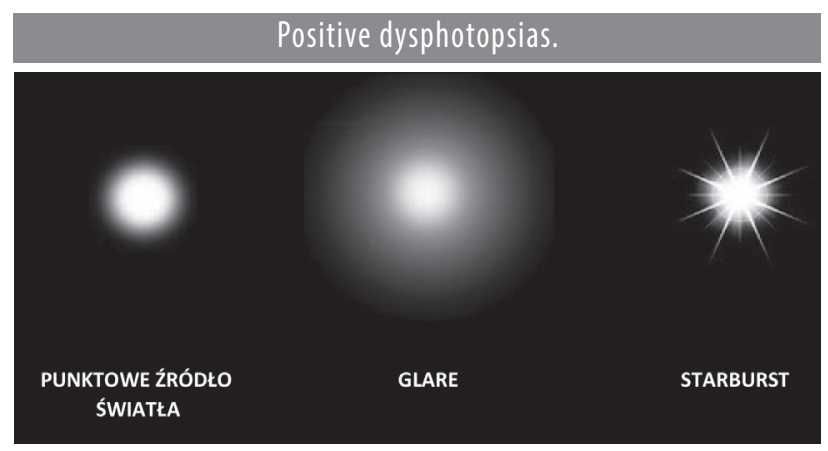

\section{OWN EXPERIENCE}

In Poland, EDoF lenses are not yet so popular among patients and ophthalmologists. However, the influence of trends in other countries has been observed, so EDoF's popularity is likely to improve. EDoF lenses also probably require a much shorter neuroadaptation time, but there are currently no studies supporting this thesis. Due to the smaller number of adverse effects, EDoF lenses seem to be a good choice for several types of patients [10]:

- Patients with contraindications on the use of multifocal lenses.

- Patients with high vision requirements due to better distance vision than with multifocal lenses, but get the additional advantages of good vision at intermediate distance than with monofocal lenses.

- Patients who spend a lot of time driving motor vehicles, especially in scotopic conditions, due to a lower risk of halo, glare, or starburst effects, while also maintaining good vision at a distance of $70-80 \mathrm{~cm}$, including of dashboards.

EDoF lenses have similar indications for use as monofocal lenses and therefore can replace monofocal lenses for patients who are not likely to be able to use multifocal lenses because of possible future contraindications.

Our experience shows that most patients are very satisfied with EDoF lenses. Far-sightedness is similar to the kind of visual acuity that can be achieved with monofocal lenses. Near-sightedness is at the level of Sn 0.5-0.75 without correction, or Sn 0.5 with correction +1.0 Dsph. Additionally, patients experience unwanted light effects much less frequently than with multifocal lenses, and if they do occur, the patients do not describe them as preventing normal functioning.

\section{CONCLUSIONS}

EDoF lenses are a relatively new but well-studied type of lens, the use of which should be considered during cataract and refractive lens replacement surgeries. A decision on what type of lens to use should be taken after thorough review of the patient's medical history, a physical examination, and an assessment of the patient's ocular needs. This should be followed by providing information to the patient about the outcomes to be expected after the procedure. EDoF lenses show few adverse effects and are well tolerated by patients. They should be considered to be better than monofocal lenses, and also a good alternative to multifocal lenses. In our opinion, EDoF lenses will come to be used more widely as ophthalmologists and patients learn more about them.

Figures: from the author's own materials. 


\section{CORRESPONDENCE}

\section{Andrzej Grąbczewski, MD}

Independent Public Clinical Ophthalmology Hospital

00-576 Warszawa, ul. Marszałkowska 24/26

e-mail: andrzejgrabczewski@live.com

\section{ORCID}

Andrzej Grąbczewski - ID - http://orcid.org/0000-0002-3445-5594

Martyna Jendrzejczyk - ID - http://orcid.org/0000-0002-8579-350X

\section{References}

1. Xu J, Zheng T, Lu Y. Comparative Analysis of Visual Performance and Astigmatism Tolerance with Monofocal, Bifocal, and Extended Depth-of-Focus Intraocular Lenses Targeting Slight Myopia. J Ophthalmol. 2020; 2020: 9283021.

2. Savini G, Schiano-Lomoriello D, Balducci N. Visual Performance of a New Extended Depth-of-Focus Intraocular Lens Compared to a Distance-Dominant Diffractive Multifocal Intraocular Lens. J Refract Surg. 2018; 34(4): 228-35. http://doi.org/ 10.3928/1081597X-20180125-01.

3. Breyer DRH, Kaymak H, AxT et al. Multifocal intraocular lenses and extended depth of focus intraocular lenses. Asia Pac J Ophthalmol (Phila). 2017; 6: 339-49.

4. Bourne RRA, Jonas JB, Bron AM. Prevalence and causes of vision loss in high-income countries and in Eastern and Central Europe in 2015: magnitude, temporal trends and projections. Br J Ophthalmol. 2018; 102(5): 575-85.

5. Ajibode HA, Fakolujo VO, Onabolu OO et al. A community-based prevalence of presbyopia and spectacle coverage in Southwest Nigeria. J West Afr Coll Surg. 2016; 6: 66-82.

6. McDonnell PJ, Lee P, Spritzer K et al. Associations of presbyopia with vision-targeted health-related quality of life. Arch Ophthalmol. 2003; 121(11): 1577-81.

7. Assi L, Chamseddine F, Ibrahim P. A Global Assessment of Eye Health and Quality of Life. A Systematic Review of Systematic Reviews. JAMA Ophthalmol. 2021; 139(5): 1-16.

8. Kanclerz P, Toto F, Grzybowski A. Extended Depth-of-Field Intraocular Lenses: An Update. Asia Pac J Ophthalmol (Phila). 2020; 9(3): 194-202.

9. Artal P, Manzanera S, Piers P et al. Visual effect of the combined correction of spherical and longitudinal chromatic aberrations. Opt Express. 2010; 18: 1637-48.

10. Savini G, Balducci N, Carbonara C. Functional assessment of a new extended depth-of-focus intraocular lens. Eye. 2018; 33(3). http:// doi.org/10.1038/s41433-018-0221-1.

Authors' contributions:

Andrzej Grąbczewski: 90\%; Martyna Jendrzejczyk: 10\%.

Conflict of interest:

None.

Financial support:

None.

Ethics:

The content presented in the article complies with the principles of the Helsinki

Declaration, EU directives and harmonized requirements for biomedical journals. 